4.

Derecho procesal 

Revista de Derecho

de la Pontificia Universidad Católica de Valparaíso

XXXII (Valparaíso, Chile, $1^{\text {er }}$ Semestre de 2009)

[pp. 347 - 362]

\section{FORMA Y SUSTANCIA EN EL RAZONAMIENTO PROBATORIO. EL ALCANCE DEL CONTROL SOBRE LA VALORACIÓN DE LA PRUEBA A TRAVÉS DEL RECURSO DE NULIDAD PENAL*}

["Form and Substance in the Probatory Reasoning. Scope of Control on the Evidence Assesment by Way of the Recourse for Annulment Regarding

Criminal Law Matters"]

\section{Daniela Accatino** \\ Universidad Austral de Chile}

\begin{abstract}
RESUMEN
El trabajo analiza la interpretación que la jurisprudencia chilena ha hecho de la causal de nulidad por omisión de alguno de los requisitos de la sentencia penal relativos a la justificación de los hechos y, en particular, examina la tendencia a distinguir entre el control formal del razonamiento probatorio y el control sustancial de la valoración realizada por el Tribunal de juicio oral, para excluir este último del ámbito legítimo del recurso. La autora sostiene que se trata de una restricción que carece de justificación jurídico-procesal, que se explica sólo por la adhesión a una interpretación subjetivista del estándar de prueba penal y que resulta contradictoria con otras prácticas de las propias Cortes que la sostienen.

Palabras clave: Prueba - Proceso penal - Razonamiento judicial.
\end{abstract}

\begin{abstract}
This article analyses the interpretation the Chilean jurisprudence has construed of the annulment causes by omission of some of the requirements of the criminal judicial ruling regarding the justification of facts and it especially examines the tendency to distinguish between the formal control of the probatory reasoning and the substantial control of the assessment carried out by the Oral Trial Court, to exclude the latter from the legitimate scope of the motion. The author states that this restriction lacks legal-procedural justification that can only be explained because it is adhered to a subjectivist interpretation of the standard of the criminal evidence which is in conflict with other practices of the Courts that sustain it.

Keywords Evidence - Criminal procedure - Legal reasoning.
\end{abstract}

* Este trabajo forma parte de una investigación que cuenta con el apoyo de FONDECYT (Proyecto No 11060129).

** Doctora en Derecho. Profesora de Teoría del Derecho, en la Facultad de Ciencias Jurídicas de la Universidad Austral de Chile. Dirección postal: Campus Isla Teja s/n, Casilla 567, Valdivia, Chile. Correo electrónico: daccatino@uach.cl. 


\section{EL NUEVO RECURSO DE NULIDAD PENAL Y LOS VIEJOS PROBLEMAS DE DESLINDES}

La historia de los recursos extraordinarios, y en particular la de la casación, es una historia de tensiones fronterizas, en la que el límite entre aquellas cuestiones que pueden y aquellas que no pueden ser objeto de conocimiento ha estado expuesto a constantes incursiones y desplazamientos. De esos viejos problemas de deslindes, posiblemente el más célebre es el que se refiere a la distinción entre hechos y derecho. Configurada la casación como un recurso dirigido a controlar la legalidad in procedendo e in iudicando de una decisión judicial, quedaban por tanto fuera de su alcance las cuestiones de hecho, entendidas como aquellas relativas a la prueba de los enunciados empíricos relevantes, excluidas las concernientes a la aplicación de normas relativas a la admisibilidad y la rendición de la prueba o de normas de prueba legal (cuestiones, estas últimas, de derecho, por referirse a la aplicación de normas) ${ }^{1}$. Conforme a ese modelo, entonces, y en el contexto de un procedimiento en el que rigiera la libertad en la valoración de la prueba, la decisión de tener por probados (o por no probados) ciertos hechos no resultaría, en principio, susceptible de control.

Sólo en principio, eso sí, pues los hechos han ido encontrando rendijas por las que infiltrarse en el templo del derecho. El control del cumplimiento de la exigencia de motivación con respecto a los enunciados probatorios ha sido posiblemente su principal caballo de Troya ${ }^{2}$. Pero también ha servido al mismo fin la revisión del respeto de la presunción de inocencia, sobre todo en su dimensión de regla de juicio, ${ }^{3}$ e incluso el control de la errónea aplicación del derecho, en tanto se considera a las máximas de la experiencia, los conocimientos científicos o los principios lógicos como normas jurídicas ${ }^{4}$. Extremando ese solapamiento entre hechos y derecho se puede incluso decir que "cuando una norma es aplicada a un

${ }^{1}$ Cfr. TARuffo, Michele, Il vertice ambiguo. Saggi sulla cassazione civile (Bolonia, Il Mulino, 1991); y Nieva Fenoll, Jorge, El hecho y el Derecho en la casación penal (Barcelona, José Ma Bosch Editores, 2000).

${ }^{2}$ Cfr. Iacoviello, Francesco, La motivazione della sentenza penale e il suo controllo in cassazione (Milano, Giuffrè, 1997), pp. 261 ss.; Bacigalupo, Enrique, La impugnación de los hechos probados en la casación penal (Buenos Aires, Editorial Ad Hoc, 1994).

${ }^{3}$ Fernández López, Mercedes, Prueba y presunción de inocencia (Madrid, Iustel, 2005), pp. 233 ss.

${ }^{4}$ Roxin, Claus, Derecho procesal penal (Buenos Aires, Editores del Puerto, 2000), pp. 470 ss. 
hecho que ha sido incorrectamente establecido existe aplicación incorrecta de la ley"s.

Aunque el recurso de nulidad penal no es asimilable a la tradicional casación, ${ }^{6}$ el lastre de un temor a la irrupción excesiva de los hechos que en ese contexto se desarrolló parece seguir pesando sobre la interpretación de su alcance ${ }^{7}$.

\section{LA DISTINCIÓN ENTRE EL CONTROL FORMAL DEL RAZONAMIENTO Y EL CONTROL SUSTANCIAL DE LA VALORACIÓN}

Ese temor a la presión invasora de las cuestiones fácticas se expresa en una tendencia, relativamente consolidada en el discurso jurisprudencial, a reducir las vías de control sobre la valoración de la prueba a la causal de falta de fundamentación de la declaración de hechos probados [artículo 374 letra e) en relación con el 342 letra c) CPP.], descartando una vía autónoma de control a través de la causal genérica del artículo 373 letra a) por infracción sustancial del derecho a la presunción de inocencia, y a interpretar restrictivamente esa causal, introduciendo una distinción entre el control formal del razonamiento probatorio y el control sustancial de la valoración realizada por el tribunal, para excluir este último del ámbito legítimo del recurso del nulidad ${ }^{8}$.

${ }^{5}$ Roxin, Claus, cit. (n. 4), pp. 471-2.

${ }^{6}$ Aunque hay quienes destacan la proximidad entre el recurso de nulidad y el de casación: cfr. por todos TAvolari, Raúl, Instituciones del nuevo proceso penal (Santiago, Editorial Jurídica, 2005), p. 202. Otros apuntan a su específico carácter amplio y desformalizado: Cortez Matcovich, Gonzalo, El recurso de nulidad. Doctrina y jurisprudencia (Santiago, LexisNexis, 2006), p. 37. Lo que interesa poner de relieve en este momento es que el recurso de nulidad se inspira en una concepción general de los recursos procesales y, en particular, en una concepción del control de legalidad distinta a la que inspiró históricamente y marcó el desarrollo de la tradicional casación.

${ }^{7}$ No es este el único lastre asociado a los avatares de la distinción entre hechos y derecho en la historia de la casación que carga el recurso de nulidad penal. También soporta -en las prácticas de decisión de algunas Cortes- el peso del error conceptual de caracterizar a la calificación jurídica de los hechos como una cuestión de valoración de la prueba, sujeta a las mismas restricciones a las que, como veremos, se encuentra sujeta ésta en sus posibilidades de revisión. Cfr. al respecto Cortez Matcovich, Gonzalo, cit. (n. 6), p. 42 ss.

${ }^{8}$ Pueden verse numerosas referencias jurisprudenciales en Cortez Matcovich, Gonzalo, cit. (n. 6), pp. 319 ss. Sólo existe registro de una sentencia (con dos votos disidentes) en la que la Corte Suprema ha accedido a controlar la suficiencia de la prueba a través de la causal de infracción sustancial de garantías (presunción de inocencia): se trata de la sentencia de 25 de abril de 2005, rol № 740-05. 
Para articular (y de paso justificar) esa restricción, se suele citar un fragmento del último informe de la Comisión de Constitución, Legislación, Justicia y Reglamento del Senado, que durante la tramitación del Código Procesal Penal-específicamente, tras proponer que se descarte el recurso extraordinario que había introducido la Cámara de Diputados para el caso que la decisión del tribunal se hubiera apartado en forma manifiesta y arbitraria de la prueba rendida en el juicio oral-, sostiene que: "Si se apreció bien o se apreció mal la prueba, por tanto, no es un aspecto que esté sujeto al control de un tribunal superior. Otra cosa es la revisión que éste puede hacer acerca de la relación lógica entre la valoración de la prueba que los sentenciadores hacen y las conclusiones a que llegan en su fallo" . Lo que resultaría vedado a las Cortes al conocer de un recurso de nulidad sería entonces el "ponderar las razones" aportadas para la justificación de la decisión sobre los hechos, revisando "si los razonamientos de los jueces a que resultan suficientemente convincentes" o si un determinado elemento de prueba "tiene por si mismo fuerza suficiente como para formar una convicción condenatoria" 10 . Y, como contrapartida, lo único que cabría controlar es "si el fallo ha cumplido o no con los requisitos formales"11.

En cuanto al alcance de ese control formal, nuestra jurisprudencia ha tendido a oscilar entre una interpretación (nuevamente) restrictiva, que admite sólo la revisión de la existencia de razones justificativas claramente expresadas en la sentencia y de la ausencia de contradicciones entre ellas, y una interpretación estricta, que, tomando en serio la remisión expresa del artículo 342 letra c) CPP. al artículo 297 del mismo cuerpo legal, admite también la revisión de la concordancia entre el razonamiento justificativo del tribunal y los principios de la lógica, las máximas de la experiencia y los conocimientos científicamente afianzados. Esta última interpretación tiende a predominar en la práctica judicial más reciente (lo que no debiera resultar sorprendente, dada la dificultad de justificar la primera de las interpretaciones a la luz de la cadena de remisiones normativas expresas antes citada) ${ }^{12}$.

9 Segundo Informe de la Comisión de Constitución, Legislación, Justicia y Reglamento, p. 1167 (disponible en: www.bcn.cl).

${ }^{10}$ Son expresiones utilizadas en la Sentencia de la Corte de Apelaciones de Rancagua, de 30 de marzo de 2004, rol No 2-2004.

${ }^{11} \mathrm{Cfr}$. varias sentencias de diversas Cortes de Apelaciones que utilizan la misma fórmula en Cortez Matcovich, Gonzalo, cit. (n. 6), pp. 336 ss.

${ }^{12}$ Existe un punto diferente que ha generado también disparidad de interpretaciones, que no se refieren directamente al alcance del control que puede efectuarse a través del recurso de nulidad, sino más bien al alcance de la exigencia de fundamentación de los enunciados probatorios. Se trata de la discusión en torno al sentido del requerimiento de "hacerse cargo en su fundamentación" de toda la prueba rendida, 
Si se asume esta última interpretación, el trazado de la frontera entre el control formal admisible y el control sustancial vedado parece coincidir con la línea que separa dos momentos analíticamente distinguibles, desde la perspectiva de una teoría general del razonamiento probatorio, que integran lo que globalmente designamos como valoración de la prueba. ${ }^{13}$ El primer momento, que podemos llamar el de la valoración en sentido estricto, designaría la determinación del apoyo empírico que los elementos de juicio aportados al proceso proporcionan a las hipótesis en conflicto, de forma individual y en conjunto. Desde el punto de vista de la justificación de los enunciados probatorios, este momento correspondería al establecimiento de relaciones lógicas de corroboración entre los enunciados fácticos que se trata de probar y los elementos de juicio disponibles, lo que supone mostrar que a la luz de nuestros conocimientos previos del mundo (en especial, de las generalizaciones empíricas aceptadas) esos elementos de juicio constituyen un evento o estado de cosas predecible si suponemos hipotéticamente que esos hechos verdaderamente han ocurrido ${ }^{14}$.

que enfrenta la tesis que entiende que ella requiere un análisis individualizado de cada medio de prueba, a aquella que sostiene en cambio que bastaría su señalamiento y su análisis global. Aunque se encuentran todavía sentencias que asumen esta última perspectiva, la primera interpretación tiende a ser predominante y ha sido sostenida reiteradamente por la Corte Suprema. Vid. al respecto, Accatino, Daniela, El modelo legal de justificación de los enunciados probatorios y su control a través del recurso de nulidad, en La Misma (editora), Problemas de formación y valoración de la prueba en el nuevo proceso penal (en prensa).

${ }^{13}$ Cfr. Ferrer Beltrán, Jordi, La valoración racional de la prueba (Madrid, Marcial Pons, 2007), pp. 41 ss., quien distingue, considerando el conjunto de la actividad probatoria en el proceso, entre tres momentos: el momento de la formación de los elementos de juicio (que comprende la actuación de los controles de admisibilidad), el momento de la valoración de la prueba y el momento de la decisión sobre los hechos probados. Una aplicación de la distinción, en la que se pone de relieve como el último momento queda comprendido también en lo que usualmente se designa como valoración de la prueba, puede verse en Accatino, Daniela, La aceptabilidad de los enunciados empiricos en el proceso penal, en Fernández, José Ángel (editor), Hacia una racionalización del derecho penal chileno. Actas de las IV Jornadas Chilenas de Derecho Penaly Ciencias Penales (Santiago, Legal Publishing, 2008).

${ }^{14}$ Esta caracterización de este primer momento del razonamiento probatorio judicial supone aceptar, en primer lugar, que éste está sujeto a los criterios generales de racionalidad propios del conocimiento empírico (un supuesto que, en el caso de nuestro sistema procesal penal, encuentra una base legal en el artículo 297 CPP, en cuanto sujeta expresamente la valoración de la prueba a los principios lógicos, las máximas de la experiencia y los conocimientos científicamente afianzados, descartando una interpretación subjetivista y potestativa de la libre valoración de la prueba: al respecto, véase: Accatino, Daniela, Convicción, justificación y verdad en la valoración de la prueba, en Anuario de Filosofía Jurídica y Social 24 (2006), pp. 39-50; y LA 
Esas relaciones de corroboración se expresan como inferencias inductivas que en ningún caso pueden demostrar la verdad de una hipótesis, sino que sólo la confirman en un cierto grado, como una posible explicación de la existencia de ese elemento de juicio. De ahí que sea necesario considerar un segundo momento en la valoración de la prueba, que podemos llamar el de decisión sobre la prueba, en el que corresponderá determinar si el apoyo inductivo aportado por los elementos de juicio disponibles a una hipótesis es suficiente para tenerla por probada en el contexto de una determinada clase de proceso. Esta cuestión podría resolverse por aplicación de los criterios generales de racionalidad, que llevarían a considerar suficiente la confirmación de una hipótesis en mayor grado que las demás hipótesis en conflicto, si para el derecho sólo fuera relevante, en este momento del juicio sobre los hechos, el fin de la averiguación de la verdad (o, en otros términos, de la reducción del error). Pero al derecho también puede interesarle la distribución del error, de modo que por la mayor gravedad de los costos de algunos de ellos, prefiera evitarlos en mayor medida que otros, estableciendo, para tener por probada esa clase de hechos, un estándar de prueba más exigente que el de la mayor corroboración con respecto a las otras hipótesis en conflicto. Esto es lo que suponemos que ocurre en el ámbito del proceso penal, donde se considera más grave desde el punto de vista de sus costos una falsa condena que una falsa absolución y se establece por eso un estándar de prueba exigente para tener por probada la acusación. Esta es la función que cumpliría el estándar de prueba “más allá de toda duda razonable”, acogido por el artículo 340 CPP. Por consiguiente, desde el punto de vista del razonamiento justificativo, este segundo momento correspondería a la justificación de la suficiencia de los elementos de juicio disponibles para declarar probada la hipótesis de la acusación, de acuerdo a los criterios fijados por el estándar de prueba ${ }^{15}$.

La distinción entre estos dos momentos en la valoración de la prueba y en la justificación de los enunciados probatorios aporta claridad a la

MISMA, La aceptabilidad, cit. (n. 13). En segundo lugar, supone admitir también que el modelo de la probabilidad lógica o inductiva, o basado en esquemas de corroboración de hipótesis, es el que mejor permite dar cuenta del razonamiento probatorio judicial, superando a otros modelos como el de la probabilidad estadística o el modelo matemático basado en probabilidades subjetivas. Una buena justificación de la preferencia por el primer modelo puede verse en CoHen, Jonathan, The Probable and the Provable (Oxford, Clarendon Press, 1977), pp. 49 ss.; Gascón Abellán, Marina, Los hechos en el Derecho (Madrid, Marcial Pons, 1999), pp. 161 ss.; y Ferrer Beltrán, Jordi, cit. (n. 13), pp. 96 ss.

${ }^{15}$ Obviamente se suscita la pregunta por la identificación de los criterios de suficiencia que fijaría el estándar de la "convicción más allá de toda duda razonable". Esta cuestión será analizada más adelante, IV. 
diferenciación jurisprudencial entre un control formal del razonamiento y un control sustancial de la valoración de la prueba. El primero apuntaría a verificar la existencia de relaciones lógicas de corroboración, correctamente establecidas en la fundamentación de la sentencia, entre los elementos de juicio aportados al proceso y los enunciados empíricos que se tienen por probados. El segundo apuntaría en cambio a controlar si se encuentra correctamente justificada o no, de acuerdo al estándar de prueba, la suficiencia (o la insuficiencia, en su caso) de los elementos de juicio para tener por probado un cierto enunciado empírico. Es esta última clase de control la que resultaría excluida por la interpretación dominante del alcance del recurso de nulidad.

Que hayamos avanzado en claridad y que la distinción jurisprudencial entre dos niveles de control resulte plausible desde el punto de vista analítico, no implica, sin embargo, que resulte justificada la restricción del alcance del control que se realiza a través del recurso de nulidad a uno de esos niveles. Para resolver esta cuestión es necesario evaluar los argumentos jurídicos que se esgrimen para sostenerla.

\section{UNA RESTRICCIÓN QUE CARECE DE JUSTIFICACIÓN JURÍDICO-PROCESAL}

El primer argumento que suele ser invocado para justificar la exclusión de un control dirigido a la suficiencia de los elementos de juicio valorados en la sentencia para tener por probado un cierto enunciado fáctico apunta a las características distintivas del recurso de nulidad y, en particular, al hecho de tratarse de un recurso de derecho estricto, que no está dirigido inmediatamente a obtener un nuevo pronunciamiento sobre aquello que fue objeto del proceso y que no constituye, por tanto, instancia. Sobre esa base se sostiene que no es posible "alterar los hechos que fueron fijados en la sentencia del Tribunal Oral" y que la Corte "carece de competencia para introducir modificaciones al establecimiento de los hechos que se hubieren dado por probados", de modo que "está impedida para declarar que no está probado lo que el tribunal a quo declaró estar probado"16.

La argumentación hasta aquí parece impecable, siempre que la entendamos referida a lo que una Corte, conociendo de un recurso de nulidad, puede decidir o, dicho de otro modo, a lo que tiene competencia para hacer en la parte dispositiva de sus sentencias. Pues efectivamente

${ }^{16}$ Se trata de expresiones contenidas, respectivamente, en sentencias dictadas por las Cortes de Apelaciones de La Serena (26 de septiembre de 2002), en Revista del Procesal Penal 3; y de Concepción (19 de julio de 2004, rol No 232-2004 y de 22 de noviembre de 2008, rol No 407-2008). 
una Corte sólo puede declarar en su fallo si es nulo o no el juicio oral y la sentencia reclamados, determinar el estado en que haya de quedar el procedimiento y ordenar la remisión de los autos al tribunal no inhabilitado que corresponda para que disponga la realización de un nuevo juicio ${ }^{17}$. Sin embargo, contra lo que el argumento habitual parece sugerir, de esta caracterización correcta de la competencia de la Corte no se sigue que ella no pueda fundar, en la parte considerativa de su sentencia, la declaración de nulidad que formula en la parte dispositiva, en la falta de justificación de la suficiencia de los elementos de juicio disponibles para tener por probado un cierto enunciado. Esa clase de argumento no resulta excluido por el carácter extraordinario o de derecho estricto del recurso, pues precisamente uno de los motivos de impugnación expresamente previstos por la ley es la omisión de alguno de los requisitos relativos a la fundamentación de los enunciados probatorios ${ }^{18}$. Ni tampoco se sigue de la negación del carácter de instancia del recurso de nulidad, pues, como sostuve más arriba, adoptar una nueva decisión sobre los hechos y revisar la justificación de una decisión sobre los hechos son cuestiones conceptualmente diferentes. Por más profundo y exhaustivo que sea este control no hay riesgo de que subrepticiamente se convierta en nueva decisión. Afirmar ese riesgo sería algo así como decir que mientras más profundo es el control que un auditor realiza sobre el balance de una sociedad, tanto más se transforma en administrador ${ }^{19}$.

El segundo argumento que es invocado habitualmente para avalar la exclusión de un control sobre la justificación de la suficiencia de la prueba tampoco resulta convincente. Él se apoya en la inmediación, que impediría que un tribunal que no ha tenido acceso personal y directo a las pruebas pueda revisar el juicio sobre la suficiencia de ellas para tener algún hecho por probado ${ }^{20}$. Si asumimos la distinción antes delineada entre decidir

${ }^{17}$ Cfr. artículos 384 y 386 CPP. Como se sabe, hay ciertos casos de errónea aplicación de derecho, previstos en el artículo 385, en los que la Corte se encuentra facultada para invalidar sólo la sentencia y dictar sentencia de reemplazo, reproduciendo las consideraciones de hecho de la sentencia anulada.

${ }^{18} \mathrm{La}$ constante referencia al carácter extraordinario del recurso de nulidad parece estar asociada de modo más general a la atribución a esa calidad de consecuencias que en rigor son por completo ajenas a ella. Es lo que ocurre también, como pone de relieve Cortez Matcovich, con la usual conexión entre ese carácter y el rigor formalista en la interpretación de los presupuestos de admisión. Cfr. Cortez Matcovich, Gonzalo, cit. (n. 6), pp. 40-42.

${ }^{19}$ Es la metáfora que sugiere IACOvielLo, Francesco, Motivazione (controllo della), en Enciclopedia Giuridica (Milano, Giuffrè, 2000), IV (“Aggiornamento”), p. 782.

${ }^{20}$ Así, por ejemplo, una sentencia de la Corte de Apelaciones de Rancagua, de 22 de noviembre de 2004, publicada en la Revista del Procesal Penal 29, señala que "Es 
sobre la suficiencia de la prueba y revisar la justificación de la suficiencia, la inmediación sólo podría representar un obstáculo para esta segunda actividad si ella fuera entendida como un método de decisión, según el cual la decisión sobre la suficiencia de la prueba acontecería en contacto inmediato con ésta, como una epifanía inefable respecto de la cual no cabría en definitiva justificación ni tampoco, por supuesto, control.

Es posible que esta concepción de la inmediación se encuentre efectivamente presente en el imaginario judicial ${ }^{21}$, posiblemente unida, como veremos en la siguiente sección, a una interpretación subjetivista del estándar de prueba. Pero eso no impide descartarla como claramente errónea. La inmediación sólo designa, junto a la oralidad y la contradicción, una técnica de formación de la prueba y su contenido específico consiste en requerir la presencia ininterrumpida de los sujetos procesales, a fin de excluir la delegación en la recepción de la prueba (y la consiguiente mediación de algún sujeto distinto al tribunal, que elevaría el riesgo de error en la transmisión de la información) y a asegurar el principio de contradicción, especialmente en el ámbito del interrogatorio y el contra-interrogatorio de testigos y peritos ${ }^{22}$.

El único efecto de la inmediación desde el punto de vista de la justificación de los enunciados probatorios consiste en hacer innecesaria la fundamentación de las constataciones, esto es, de aquello que fue directamente percibido por el tribunal (por ejemplo, que el testigo $\mathrm{T}$ declaró haber visto a Ticio saliendo de la casa de Caio a las 01:15 AM del día 25 de noviembre), pues la comprobación empírica directa basta en estos casos como justificación. Ella no excluye en cambio, en absoluto, la justificación de su valoración como elemento de juicio a favor de un cierto enunciado (en el ejemplo anterior, la valoración del testimonio de T como elemento de juicio que corrobora la hipótesis según la cual Ticio salió de la casa de Caio a las 13.15 AM del día 25 de noviembre y la valoración

propio del proceso oral, por imperativo del principio de inmediación, que sea el Tribunal ante el cual se realizó el juicio el que valore soberanamente la prueba, y por tanto la credibilidad de los declarantes. El Tribunal superior, más allá de que le parezca acertada o no esa valoración; más allá de que comparta o no las conclusiones, no puede alterarla, porque no presenció el juicio y su mirada es necesariamente mediata. Ese es el basamento del sistema reformado de procedimiento penal [...]" (p. 70). Otros ejemplos pueden verse en Cortez Matcovich, Gonzalo, cit. (n. 6), pp. 321 ss.

${ }^{21}$ Es lo que sugieren expresiones del tipo "el sentenciador sólo puede fallar de acuerdo con las impresiones personales que obtenga de los medios de prueba" (sentencia de la Corte de Apelaciones de Concepción, de 27 de octubre de 2008, rol № 407-2008)

${ }^{22}$ Cfr. Andrés IbáŃEz, Perfecto, Sobre el valor de la inmediación (una aproximación crítica), en Jueces para la Democracia 48 (2003), pp. 57-66. 
de su suficiencia para tener por probada esa hipótesis) ni el control de esa justificación. $^{23}$

Resta por analizar el argumento que dice relación con la historia legislativa del Código Procesal Penal y en particular con la supresión, en la discusión que tuvo lugar en el Senado, del recurso extraordinario que había propuesto incorporar la Cámara de Diputados para obtener la repetición del juicio oral cuando el tribunal se hubiese apartado en forma manifiesta y arbitraria de la prueba rendida. Recordemos que es en ese contexto -y con el objeto de destacar las diferencias y ventajas del nuevo recurso de nulidad- que el segundo informe de la Comisión de Constitución formula la advertencia que es frecuentemente citada cuando se introduce la distinción que estamos examinando y según la cual "si se apreció bien o se apreció mal la prueba no es un aspecto que pueda estar sujeto al control de un tribunal superior".

Los argumentos que la Comisión de Constitución ofrece en su Segundo Informe para descartar la propuesta del recurso extraordinario y proponer un nuevo recurso de nulidad que reemplazara tanto a aquél como al recurso de casación inicialmente contemplado en el proyecto, apuntan, en primer lugar, a evitar el riesgo de una recarga indebida del sistema dada la posibilidad que "so pretexto de que el tribunal se ha apartado manifiestay arbitrariamente de la prueba rendida, se pida habitualmente que la Corte de Apelaciones revise los registros del juicio oral' 24 . Por otra parte, se sostiene que de ese modo se evita una incongruencia con el rechazo legislativo del recurso de apelación, que supone que "el tribunal de juicio oral, que es el que recibió la prueba, es el más idóneo para valorarla y dictar la sentencia" 25 . Por esas razones se prefiere, agrega el Informe más adelante, recoger "con mayor propiedad" la inquietud de la Cámara de Diputados a través de la causal de falta de fundamentación de las conclusiones acerca de los hechos y circunstancias que se dieren por probados; una vía, se señala, que se compadece mejor con el hecho que los jueces de juicio oral "tienen la facultad de apreciar libremente la prueba, salvo ciertos limites que tienen que ver con las reglas de la lógica formal, las máximas de experiencia y los conocimientos cientificamente afianzados" 26 .

Sin perjuicio de las debilidades que afectan en general a los argumentos interpretativos que pretenden determinar la voluntad del legislador y de

\footnotetext{
${ }^{23}$ Sobre los malos entendidos a que en esta materia da lugar la ambigüedad en el uso de la distinción entre prueba directa y prueba indirecta cfr. GASCÓn Abellán, Marina, cit. (n. 14), pp. 86 ss.

${ }^{24}$ Segundo Informe, cit. (n.9), p. 1105.

${ }^{25}$ Segundo Informe, cit. (n.9), p. 1105.

${ }^{26}$ Segundo Informe, cit. (n.9), p. 1167.
} 
que algunas de las opiniones reseñadas puedan -especialmente la última de ellas, según veremos en el próximo apartado- considerarse equivocadas a la luz de otras disposiciones del Código Procesal Penal, hay a mi juicio una forma de entender el sentido de la sustitución del recurso extraordinario por la causal de nulidad por falta de fundamentación de los enunciados probatorios, que excluye que ella pueda justificar la expulsión de la justificación de la suficiencia de la prueba del ámbito legítimo del control a través del recurso de nulidad. Se trata de poner de relieve como lo que se sustituye es, en definitiva, un recurso que tenía por objeto la evaluación directa de la suficiencia de la prueba para condenar a la luz de los registros del juicio oral, por otro que tiene por objeto el control de la justificación de las conclusiones a las que el tribunal de juicio oral llega acerca de la prueba. Lo que la Corte examina en el nuevo modelo de recurso es la fundamentación de la sentencia del tribunal de juicio oral, pudiendo tomar en cuenta los registros del juicio sólo si se denuncia la falta de consideración de alguna prueba rendida. Desde esta perspectiva no hay ninguna razón para negar que el control de la fundamentación se extienda también a la justificación de la suficiencia de la prueba. No se le pide entonces a la Corte que valore de nuevo la prueba a la que accede a través del registro del juicio oral y compare su propia valoración con la que ha llevado al Tribunal de juicio oral a condenar o absolver, para determinar, parafraseando el Informe de la Comisión, "si se apreció bien o se apreció mal la prueba”. Lo que se le pide en ese caso es que examine si se encuentra justificada la valoración de la prueba como suficiente para condenar o insuficiente para absolver.

\section{UNA RESTRICCIÓN SÓLO EXPLICABLE POR UNA INTERPRETACIÓN SUBJETIVISTA DEL ESTÁNDAR DE PRUEBA PENAL}

Hay otro argumento que también se suele ofrecer cuando se recurre a la distinción entre control formal y control sustancial y se niega lugar a la revisión de la justificación de la suficiencia de la prueba. He reservado su análisis hasta ahora porque, aunque carece, como los examinados anteriormente, de fuerza justificativa, resulta sin embargo significativa como explicación de esa restricción y como indicación del camino que es necesario recorrer para superarla.

La siguiente cita ofrece un buen ejemplo de esa argumentación: " $\mathrm{La}$ convicción del tribunal, más allá de toda duda razonable, sobre la perpetración de un cuasidelito, es un asunto radicado en el fuero interno de los dirimentes llamados a conocer de una determinada materia, sin que esta Corte tenga la facultad de anular por ese motivo, el juicio que ellos han sido llamados a resolver, ya que dicha decisión emana del ámbito de su propia 
conciencia, previo un conocimiento exhaustivo y directo de los hechos" 27 . El complemento habitual vendrá dado por la referencia a la "plena libertad" o "soberanía" de los jueces de juicio oral para valorar los medios de prueba, con el sólo límite de no contradecir los principios de la lógica, las máximas de la experiencia y los conocimientos científicamente afianzado ${ }^{28}$.

Esta forma de argumentar niega al estándar de prueba penal toda función normativa, al interpretarlo como una mera remisión al estado mental de convicción del juzgador. Bajo este supuesto, la resistencia a controlar la justificación de la suficiencia de la prueba se explica perfectamente. Claro, cómo vamos a controlarla si lo que el estándar de prueba dice es que es suficiente la prueba que el tribunal de juicio oral cree que es suficiente.

El problema de esta interpretación subjetivista es que deja trunco el modelo legal de justificación de los enunciados probatorios, pues aunque se acepte que hay criterios racionales para establecer las relaciones de corroboración entre los elementos de prueba y los enunciados acerca de cuya prueba se discute, la determinación de su suficiencia para tenerlos por probados queda abandonada a una suerte de epifanía inefable que acontece al juzgador. Si subsiste este enclave subjetivista -en el que resuena además la concepción de la inmediación como método de decisión-, poco es lo que se gana entonces con los alardes sobre la adhesión de nuestro sistema de valoración de la prueba a un modelo de valoración racional o de sana crítica, dado que el juicio sobre la suficiencia de la prueba queda en definitiva liberado de toda exigencia de justificación racional. ${ }^{29}$

Esta interpretación subjetivista del estándar de prueba más allá de toda duda razonable ha resultado favorecida por el desafortunado uso que el artículo 340 CPP. hace de la expresión "convicción”, así como por la lectura igualmente subjetivista de la misma fórmula que ha predominado en los sistemas jurídicos de tradición anglosajona -de donde se nos ha ocurrido importarla- y que ha sido acogida también por nuestra doctrina ${ }^{30}$.

${ }^{27}$ Sentencia Corte Suprema, 13 de enero de 2005, citada por Cortez Matcovich, Gonzalo, cit. (n. 6), p. 353.

${ }^{28}$ Pueden verse varias citas de sentencias en Cortez Matcovich, Gonzalo, cit. (n. 6), pp. 334 ss.

${ }^{29}$ En este sentido tiene razón Coloma cuando detecta una contradicción entre el artículo 297 CPP, que establecería un criterio racional de valoración de la prueba, y el artículo $340 \mathrm{CPP}$, que fijaría en cambio un criterio puramente subjetivo. Como veremos, en mi opinión la contradicción puede disolverse a través de una reconstrucción no subjetivista del estándar de prueba más allá de toda duda razonable. Cfr. CoLOMA, Rodrigo, Panorama general de la prueba en el juicio oral chileno, en El Mismo (editor), La prueba en el nuevo proceso penal (Santiago, LexisNexis, 2003), pp. 26 ss.

${ }^{30}$ Sobre las interpretaciones subjetivista en el contexto anglosajón, cfr. LaUdAN, Larry, Porqué un estándar de prueba subjetivo y ambiguo no es un estándar, en Doxa 
No estamos, sin embargo, forzados a quedarnos de brazos cruzados y aceptar esta versión subjetivista del estándar de prueba penal. En la discusión teórica contemporánea sobre la prueba judicial se encuentran esfuerzos interesantes de articulación de estándares objetivos de prueba ${ }^{31}$, que tienen en común el formularse en el lenguaje de la corroboración de hipótesis, que aplica, según hemos visto más arriba, los criterios generales de racionalidad al ámbito de la valoración de la prueba ${ }^{32}$. Ya sabemos que esos criterios no pueden determinar por sí solos un estándar de prueba capaz de distribuir los posibles errores judiciales -esa es una decisión política que debe adoptar la sociedad-, pero, una vez adoptada esa decisión, el lenguaje de la probabilidad inductiva ofrece herramientas para definir en términos objetivos el umbral más exigente de corroboración que debe satisfacer una hipótesis para poder ser aceptada como probada en un proceso penal.

Si damos por supuesto que la disposición legal que consagra el estándar de prueba más allá de toda duda razonable expresa esa decisión política de evitar con mayor intensidad las falsas condenas que las falsas absoluciones, entonces será posible utilizar el lenguaje de la corroboración (en la línea de los trabajos teóricos ya citados) para articular criterios objetivos que lo precisen, delineando una teoría de la razonabilidad de la duda. Así, podría sostenerse que ese estándar requiere, para condenar ${ }^{33}$ :

i) Que la hipótesis sea capaz de explicar los datos probatorios disponibles y que las predicciones de nuevos datos que la hipótesis permitía formular hayan sido efectivamente constatadas. Este primer nivel apunta a la riqueza de los elementos de juicio que corroboran la hipótesis y requiere una comparación entre las pruebas efectivamente aportadas y las

28 (2005), pp. 95-113; y El MISmo, Truth, Error, and Criminal Law (Cambridge, Cambridge University Press, 2006), pp. 29 ss. En cuanto a la adhesión de la doctrina procesal penal a una interpretación subjetivista, véase, por todos: HoRwitz, María Inés - López, Julián, Derecho procesal penal chileno (Santiago, Editorial Jurídica de Chile, 2004), II, especialmente pp. 162 y ss., quienes terminan por asimilarla al “estado subjetivo de certeza" (p. 163). Es interesante notar que en los sistemas de tradición anglosajona los controles de racionalidad en la valoración de la prueba no operan a través de una exigencia de justificación racional (sobre todo cuando el "traier of fact" es un jurado) sino preventivamente a través de abundantes y sofisticadas reglas de exclusión de prueba que, por ejemplo, consideran en principio inadmisibles los testigos de oídas. Cfr. al respecto Damaska, Mirjan, Evidence Law Adrift (New Haven, Yale University Press, 1997).

${ }^{31}$ Cfr. Laudan, Larry, Porqué, cit. (n. 30); El mismo, Truth, cit. (n. 30), pp. 63 ss.; Stein, Alex, Foundations of Evidence Law (Oxford, Oxford University Press, 2005), pp. 172 ss.; Ferrer Beltrán, Jordi, cit. (n. 13), pp. 139 ss.

${ }^{32}$ Véase la n. 14.

${ }^{33}$ La formulación que se presenta es próxima a la que ofrece Ferrer Beltrán, Jordi, cit. (n. 13), pp. 147 ss. 
que, resultando predecible su existencia en el caso concreto, no han sido, sin embargo, producidas.

ii) Que se hayan descartado todas las demás hipótesis compatibles con la inocencia del acusado, excepto las hipótesis ad hoc que no son empíricamente contrastables.

No pretendo justificar detenidamente en este trabajo esta formulación del estándar de prueba penal a través de criterios objetivos (que no dependen de la creencia o convicción del juzgador) ${ }^{34}$, lo que me interesa sobre todo es sugerir que se trata de un camino que es posible recorrer. Esto es importante porque, como veremos en las siguientes secciones, hay otras razones adicionales para no resignarnos a la interpretación subjetivista de ese estándar y a la distinción entre los dos niveles de control de la fundamentación de los enunciados probatorios que en ella se apoya.

\section{UNA RESTRICCIÓN NEGADA EN LAS PROPIAS PRÁCTICAS DE DECISIÓN DE LAS CORTES DE APELACIONES}

La primera de esas razones surge de la constatación de una contradicción al interior de la concepción dominante acerca del alcance del control que puede ejercerse sobre la justificación de la decisión sobre los hechos a través del recurso de nulidad. En un primer nivel esa contradicción tiene lugar porque las mismas Cortes que se resisten a controlar la justificación de la suficiencia de la prueba, tienden, en general, a exigir, para dar lugar a la nulidad de la sentencia y del juicio oral por omisión de algún requisito de fundamentación, que se establezca la influencia del defecto en la parte dispositiva de la sentencia, haciendo aplicable a dicha causal lo dispuesto por el artículo $375 \mathrm{CPP}$. En ese caso, lo que están requiriendo que se establezca es que subsanada la omisión o defecto en la valoración de algún dato probatorio, el grado de corroboración de la hipótesis de la acusación podría disminuir hasta ser insuficiente para tenerla por probada (si se trata de una sentencia condenatoria, que la ha tenido por probada) o aumentar hasta ser suficiente para tenerla por probada (si se trata de una sentencia absolutoria que no la ha tenido por probada).

Veamos los términos en que se expresan dos sentencias que valoran la concurrencia de la exigencia. La primera reza: "[...] la falta de ponderación legal de los antecedentes referidos, por parte de los sentenciadores, ha influido en los dispositivo del fallo, al ser evidente que la adecuada valoración podría conducir a la condena de todos o parte de los acusados, aun cuando esta aseveración no puede calificarse de concluyente" ${ }^{35}$. La segunda expresa: "[...] en el caso en

\footnotetext{
${ }^{34}$ Vid. Accatino, Daniela, El modelo legal, cit. (n. 12).

${ }^{35}$ Sentencia de la Corte de Apelaciones de Concepción, de 24 de marzo de 2008,
} 
examen, la causal de nulidad hecha valer reviste sólo un carácter formal, sin que aparezca o se deduzca de los términos en que ha sido planteada que haya tenido o podido tener influencia en la decisión del tribunal $y$, de ser asi, en qué magnitud. En efecto, el recurso no dice que el juez debió haber establecido como efectivos hechos diferentes a los que imputó al condenado el Ministerio Público, o que lo aseverado en el requerimiento no correspondía a la verdad de lo ocurrido, o que en el caso a juzgar concurrieran circunstancias que debieran haber llevado al tribunal a absolver, o bien a disminuir o morigerar la pena u otorgar algún beneficio para su cumplimiento. En suma, según los términos en que se plantea el recurso, no se divisa cómo una correcta fundamentación de la sentencia hubiera producido una variación en la decisión final, sobre la base de hechos que la defensa no ha controvertido ni impugnado"36.

Pero, ¿esta clase de juicios hipotéticos no suponen necesariamente valorar la suficiencia o insuficiencia de los demás elementos de juicio disponibles a fin de resolver sobre la posible relevancia de los elementos de juicio afectados por la omisión o defecto? Paradójicamente entonces, el mismo nivel de control vedado en algunos casos, resultaría exigido en cambio en otros. Inevitablemente surge la sospecha de que ocurra lo que Pastor afirma con respecto a la separación entre cuestiones de hecho y de derecho en el contexto del recurso de casación, cuando sostiene que se trata de una distinción que "sólo subsiste al servicio de una arbitrariedad judicial que no distingue hecho de derecho, ni cuestión revisable de cuestión irrevisable, sino que separa únicamente lo que quiere revisar de lo que no quiere" 37 .

[Recibido el 2 de diciembre de 2008 y aprobado el 9 de marzo de 2009].

\section{BIBLIOGRAFÍA}

AcCatino, Daniela, Convicción, justificación y verdad en la valoración de la prueba, en Anuario de Filosofía Jurídica y Social 24 (2006), pp. 39-50.

Accatino, Daniela, El modelo legal de justificación de los enunciados probatorios y su control a través del recurso de nulidad, en LA Misma (editora), Problemas de formación y valoración de la prueba en el nuevo proceso penal (en prensa).

Accatino, Daniela, La aceptabilidad de los enunciados empiricos en el proceso penal, en Fernández, José Ángel (editor), Hacia una racionalización del derecho penal

rol No 59-2008.

${ }^{36}$ Sentencia de la Corte de Apelaciones de Valparaíso, de 11 de enero de 2004, rol No 880-04.

${ }^{37}$ Pastor, Daniel, La nueva imagen de la casación penal (Buenos Aires, Editorial Ad Hoc, 2001), pp. 119-120. 
chileno. Actas de las IV Jornadas Chilenas de Derecho Penaly Ciencias Penales (Santiago, Legal Publishing, 2008).

Andrés IbáÑEz, Perfecto, Sobre el valor de la inmediación (una aproximación crítica), en Jueces para la democracia 48 (2003), pp. 57-66.

Bacigalupo, Enrique, La impugnación de los hechos probados en la casación penal (Buenos Aires, Editorial Ad Hoc, 1994).

Cohen, Jonathan, The probable and the Provable (Oxford, Clarendon Press, 1977).

Coloma, Rodrigo, Panorama general de la prueba en el juicio oral chileno, en El Mismo (editor), La prueba en el nuevo proceso penal (Santiago, LexisNexis, 2003).

Cortez Matcovich, Gonzalo, El recurso de nulidad. Doctrina y jurisprudencia (Santiago, LexisNexis, 2006)

Damaska, Mirjan, Evidence Law Adrift (New Haven, Yale University Press, 1997).

Fernández López, Mercedes, Prueba y presunción de inocencia (Madrid, Iustel, 2005).

Ferrer Beltrán, Jordi, La valoración racional de la prueba (Madrid, Marcial Pons, 2007).

Gascón Abellán, Marina, Los hechos en el derecho (Madrid, Marcial Pons, 1999).

Horwitz, María Inés - López, Julián, Derecho procesal penal chileno (Santiago, Editorial Jurídica de Chile, 2004).

IACOviello, Francesco, La motivazione della sentenza penale e il suo controllo in cassazione (Milano, Giuffrè, 1997).

IACoviello, Francesco, Motivazione (controllo della), en Enciclopedia Giuridica (IV Aggiornamento, Mián, Giuffrè, 2000).

Laudan, Larry, Porqué un estándar de prueba subjetivo y ambiguo no es un estándar, en Doxa 28 (2005), pp. 95-113.

Laudan, Larry, Truth, Error, and Criminal Law (Cambridge, Cambridge University Press, 2006).

Nieva Fenoll, Jorge, El hecho y el Derecho en la casación penal (Barcelona, José Ma Bosch Editores, 2000).

PAstor, Daniel, La nueva imagen de la casación penal (Buenos Aires, Editorial Ad Hoc, 2001).

Roxin, Claus, Derecho procesal penal (Buenos Aires, Editores del Puerto, 2000).

Stein, Alex, Foundations of Evidence Law (Oxford, Oxford University Press, 2005).

TARUfFo, Michele, Il vertice ambiguo. Saggi sulla cassazione civile (Bolonia, Il Mulino, 1991).

TAVOlari, Raúl, Instituciones del nuevo proceso penal (Santiago, Editorial Jurídica, 2005). 